\title{
Kondo effect in d-wave superconductors
}

\author{
Anatoli Polkovnikov ${ }^{\circledast}$ \\ Department of Physics, Yale University \\ P.O. Box 208120, New Haven, CT 06520-8120, USA
}

\begin{abstract}
We present theoretical investigation of a single magnetic impurity in a d-wave superconductor using the large $\mathrm{N}$ limit. It is shown that the Kondo screening occurs only in the presence of the particle-hole asymmetry. We find analytical expressions for the Kondo temperature, magnetic susceptibility and scattering matrix near the phase transition. The results are generalized for the density of states vanishing with an arbitrary exponent. Also we briefly study the modifications of the theory for the case of a non-magnetic impurity which induces a staggered spin configuration on the nearby copper atoms.
\end{abstract}

\section{INTRODUCTION}

Recent experiments in d-wave BSCCO superconductors showed some interesting phenomena in the presence of impurities on copper sites. In particular, STM showed a strong variation of the electron density of states (DOS) near $\mathrm{Ni}$ [1], 2 and $\mathrm{Zn}$ [3] atoms in $\mathrm{Bi}_{2} \mathrm{Sr}_{2} \mathrm{CaCu}_{2} \mathrm{O}_{8+\delta}$. Using NMR Kondo-like effect was in cuprates doped by Zn, $\mathrm{Ni}$ or Li impurities both above and below $T_{c}$ [4, 5, 6, , 7, 8, and an uncompensated spin $S=1 / 2$ appeared near the $\mathrm{Zn}$ impurity [9]. Although it is tempting to explain the tunneling experiments by the pure potential scattering [10], there are several key features which are not easily justified within this model (see e.g. [11]). For example, the low frequency resonance can be achieved only for a large non-universal value of the potential coupling $\sqrt{12}$. On the other hand, the Kondo screening gives a natural low energy scale and the results appear to be much more robust with respect to the variation of the composition and coupling constants 13 . We note that using STM data alone it is not possible to distinguish between the potential and Kondo scattering mechanisms leading to the variations of the DOS. The screened impurity acts as a spin singlet and is therefore similar to the potential scatterrer. However the unitarity limit in the Kondo case occurs at the coupling slightly exceeding the critical value, which is not necessarily large. The other important advantage of the Kondo mechanism is that it simultaneously explains NMR and STM measurements.

The cuprates are unconventional superconductors with linearly vanishing density of states near the four nodes. The usual Kondo picture is not valid there 14, 15, 16, 17, 18, 19. In particular, there is no phase transition if the spin-spin coupling is relatively small so the impurity remains effectively decoupled from quasiparticles. If the DOS vanishes at the Fermi surface slower than the square root of energy: $\rho(\varepsilon) \propto|\varepsilon|^{r}$ with $r<1 / 2$, then it is possible to develop a perturbation theory [14], which

*Electronic address: anatoli.polkovnikov@yale.edy; URL: http:// pantheon.yale.edu/ asp28 predicts Kondo transition at the spin-spin coupling larger than a certain critical value proportional to $r$. However if $r \geq 1 / 2$ this simple picture doesn't work. Thus both numerical renormalization group (NRG) 20, 21] and dynamic large $\mathrm{N}$ multichannel approach 22 showed that the Kondo phase exists only if the Hamiltonian doesn't respect the particle-hole symmetry. In the symmetric case the spin-spin coupling always flows to zero. On the other hand, in 24] it was argued that if the particle-hole symmetry is preserved, then there is no screening at the level of the total impurity susceptibility $T \chi_{i m p} \rightarrow 1 / 4$ at $T \rightarrow 0$, but still there is a quenching of the impurity spin and $T \chi_{l o c} \rightarrow 0$ in the zero temperature limit.

The model Hamiltonian for a pure superconductor to be exploited in the present paper is:

$$
H_{0}=\sum_{\mathbf{k}} \psi_{i}^{\dagger}(\mathbf{k})\left(\varepsilon_{\mathbf{k}} \tau_{z}^{i j}+\Delta_{\mathbf{k}} \tau_{x}^{i j}\right) \psi_{j}(\mathbf{k}),
$$

where $\varepsilon_{\mathbf{k}}=-\varepsilon_{0}\left(\cos k_{x}+\cos k_{y}\right)-\mu$ and $\Delta_{\mathbf{k}}=\Delta_{0}\left(\cos k_{x}-\right.$ $\left.\cos k_{y}\right)$ are the kinetic energy and superconducting gap respectively, $\psi(k)=\left(c_{\uparrow}(k), c_{\downarrow}^{\dagger}(-k)\right)$ is the Gor'kov-Nambu spinor. The interaction with a single impurity can be expressed in the Coqblin-Schrieffer form 25]:

$$
\begin{aligned}
& H_{i n t}=U \psi_{i}^{\dagger}\left(\mathbf{r}_{0}\right) \tau_{z}^{i j} \psi_{j}\left(\mathbf{r}_{0}\right) \\
& -\frac{J}{2}\left(\psi_{\uparrow}^{\dagger}\left(\mathbf{r}_{0}\right) F_{\uparrow}-F_{\downarrow}^{\dagger} \psi_{\downarrow}\left(\mathbf{r}_{0}\right)\right)\left(F_{\uparrow}^{\dagger} \psi_{\uparrow}\left(\mathbf{r}_{0}\right)-\psi_{\downarrow}^{\dagger}\left(\mathbf{r}_{0}\right) F_{\downarrow}\right),
\end{aligned}
$$

where $U$ and $J$ are the potential and spin-spin coupling constants, $F$ is the effective impurity spinor in the Nambu representation (it is related to the creation and annihilation operators by $\left.F_{\uparrow}=f_{\uparrow}, F_{\downarrow}=f_{\downarrow}^{\dagger}\right)$. The single impurity occupancy corresponds to the condition:

$$
f_{i}^{\dagger} f_{i}=1 \quad \Leftrightarrow \quad F_{i}^{\dagger} \tau_{z}^{i, j} F_{j}=0 .
$$

In fact, there is an additional potential like scattering term proportional to $J$ in (2), however, it disappears in the path-integral formulation of the problem [16]. If $\mu=0$ then the Hamiltonian (1) respects the particle hole symmetry, i.e. under the transformation $k_{x} \rightarrow$ $k_{x}+\pi, k_{y} \rightarrow k_{y}+\pi$ both the kinetic energy $\varepsilon_{k}$ and 
the order parameter $\Delta_{k}$ change their signs. This additional symmetry is not generic, it disappears if either chemical potential or second neighbor hopping becomes not zero. However this notion is very useful, since in many cases the particle-hole asymmetry can be treated as a perturbation. The model above but without the potential scattering term was already examined by $\mathrm{C}$. Cassanello and E. Fradkin in [16, 17. The authors concluded that there is a Kondo phase transition above some critical spin-spin coupling $J_{c}$. They found that for the linear DOS, the Kondo temperature vanishes exponentially near the phase transition. Also it was argued that in the Kondo phase the impurity susceptibility vanishes at $T \rightarrow 0$ as $T \ln 1 / T$. However, these results were obtained even in the particle-hole symmetric case. We find similar dependences of the Kondo temperature and susceptibility only if the particle-hole symmetry is broken, e.g. by non-zero potential scattering. The reason in such a discrepancy is that the symmetrization of the action with respect to positive and negative frequencies used in 16, 17 is not valid, which resulted in essential inconsistencies there. We come back to this point in the next section. The other difference between our approaches is that we start from the Hamiltonian (1) instead of its linearized nodal version [16, 17]. This is important for the analysis because (i) the particle-hole asymmetry $(U \neq 0$ or $\mu \neq 0$ ) is essentially a high energy effect, (ii) the spatial structure of the scattering can not be reproduced from the nodal version of the Hamiltonian, (iii) the expressions for the $T$-matrix and Green functions are much more apparent in the original Gor'kov-Nambu representation than in that of the nodal particles. Although at the later stage we use linearized version of the Hamiltonian for the sake of simplicity and analyticity, the results can be easily generalized for the arbitrary spectrum of quasiparticles [13].

We also study in detail the phase diagram at finite temperature, where the effects of particle hole asymmetry become crucial. The model is generalized to the case of an arbitrary exponent $r$ larger than $1 / 2$, where the simple Withoff-Fradkin picture [14 is invalid. We would also mention that the problem addressed here but for the Anderson magnetic impurity was studied in 18] using the slave-boson mean field approach. The impurity potential had been chosen in such a way that only zero or single occupancy was allowed. As a result the Hamiltonian was strongly particle-hole asymmetric from the beginning. It was found that this model predicts the Kondo effect and the particle hole symmetry of the pure superconductor is not important. The model also results in a low-energy resonance in the DOS and is consistent with the experimental spatial dependence.

The paper is organized as follows. In Section II we solve the saddle point equations and study the phase diagram in the space of the potential and spin-spin couplings $U$ and $J$. It is found that the transition to the Kondo phase occurs only in the particle-hole asymmetric case. At zero temperature the critical spin-spin coupling
$J_{c}$ saturates as $U \rightarrow 0$ if $r \leq 1$ and diverges as $1 / \sqrt{U}$ if $r>1$. In the case of a d-wave superconductor $(r=1)$, the Kondo temperature is found to vanish exponentially near $J=J_{c}$ in agreement with [17]. At the fixed temperature and for $r \leq 1$ the crossover from the high temperature $\left(T \gg T_{K}\right)$ to the low temperature $\left(T \ll T_{K}\right)$ limit occurs at $J$ diverging as $U \rightarrow 0$.

In Section III we examine the expressions for the local and total magnetic susceptibilities of the impurity and the quasiparticle density of states. It is shown that in the particle-hole symmetric case for $r \leq 1$ and $J>J_{c}$ the local susceptibility diverges as $T \rightarrow 0$, but $T \chi_{l o c} \rightarrow 0$, while $T \chi_{i m p} \rightarrow$ const. In the Kondo phase $\chi_{l o c}$ saturates and $\chi_{i m p} \rightarrow 0$. Both $\chi_{l o c}$ and $\chi_{i m p}$ as functions of temperature have a maximum at $T \approx T_{K}$. In the end of the section we derive the expression for the induced quasiparticle DOS near the impurity. It is shown that in the Kondo phase the effective scattering matrix has a pole at the frequency close to the Kondo temperature $\left(\Omega \approx T_{K}\right)$. The crucial difference between this model and simple potential scattering [10] is that the unitarity limit here occurs at finite magnitude of the spin-spin coupling $J \approx J_{c}$, which doesn't have to be large.

Section IV is devoted to the discussion of the modifications of the model for the case of a non-magnetic impurity, which induces a staggered magnetization nearby. This study is motivated by the NMR experiments [4, 9] unambiguously showing existence of the magnetic moments in the vicinity of the $\mathrm{Zn}$ atom, which substitutes $\mathrm{Cu}$. From the theoretical point of view this issue is interesting, since the spin-spin interaction becomes non-local. Assuming that the effective spin is induced on the first $\mathrm{Cu}$ neighbors of $\mathrm{Zn}$ we show that the action has four different saddle points, and for the whole range of coupling constants the configuration with the $D$ symmetry of the order parameter corresponds to the lowest energy. The critical spin-spin coupling for the non-local spin is found to decrease with $U$ as opposed to the local spin case. The spatial dependence of the induced DOS is also different. It is characteristic for the scattering on the four rather than the single site and gives an excellent agreement with the STM experiments [3].

\section{SADDLE POINT APPROXIMATION FOR A SINGLE MAGNETIC IMPURITY}

After the standard Hubbard Stratonovich decoupling and integrating out Fermion fields the partition function for the system with the Hamiltonian given by (11) and (2) becomes a functional integral over the auxiliary fields $\phi$ and $\epsilon$ with the action:

$$
\begin{aligned}
S= & -\operatorname{Tr} \ln \left(\frac{\partial}{\partial \tau}+\epsilon(\tau) \tau_{z}-\phi(\tau) G\left(\frac{\partial}{\partial \tau}\right) \phi(\tau)\right) \\
& +\frac{2}{J} \int_{0}^{\beta} \phi(\tau)^{2} d \tau
\end{aligned}
$$


where trace is taken over all antiperiodic functions of $\tau$ $(f(\tau+\beta)=-f(\tau)) ; G(\partial / \partial \tau) \equiv G\left(\mathbf{r}_{0}, \mathbf{r}_{0}, \partial / \partial \tau\right)$ is the quasiparticle Green function in the presence of the potential scattering on the impurity:

$$
G\left(\mathbf{r}, \mathbf{r}^{\prime}, \omega_{n}\right)=G^{0}\left(\mathbf{r}-\mathbf{r}^{\prime}, \omega_{n}\right)-G^{0}\left(\mathbf{r}-\mathbf{r}_{0}, \omega_{n}\right) U \tau_{z}\left(1+U \tau_{z} G^{0}\left(0, \omega_{n}\right)\right)^{-1} G^{0}\left(\mathbf{r}_{0}-\mathbf{r}^{\prime}, \omega_{n}\right),
$$

and $G^{0}$ is the free electron Green function, which in the momentum space is equal to:

$$
G_{\mathbf{k}}^{0}(\omega)=\left(-i \omega+\varepsilon_{\mathbf{k}} \tau_{z}+\Delta_{\mathbf{k}} \tau_{x}\right)^{-1} .
$$

The Lagrange multiplier $\epsilon$ in (बi) enforces the single occupancy constraint. In general $\phi$ is a complex field, however its phase can be reabsorbed into $\epsilon$ by a simple gauge transformation 23].

It is not hard to show that in a d-wave superconductor the free particle Green function at $\mathbf{r}=0$ is expressed as 10]:

$$
G^{0}(0, \omega)=G_{0}^{0}(\omega)+G_{1}^{0}(\omega) \tau_{z}
$$

with $G_{0}^{0}$ and $G_{1}^{0}$ being even and odd functions of frequency, respectively. If the free Hamiltonian is also invariant under the particle-hole transformation then $G_{1}^{0}(\omega) \equiv 0$. For simplicity we assume this is the case. In fact, it can be shown that the models with $U \neq 0$ and $G_{1}^{0} \neq 0$ can be mapped to each other. Explicitly $G_{0}^{0}\left(\omega_{n}\right)$ is given by

$$
G_{0}^{0}\left(\omega_{n}\right)=\sum_{\mathbf{k}} \frac{i \omega_{n}}{\omega_{n}^{2}+\varepsilon_{\mathbf{k}}^{2}+\Delta_{\mathbf{k}}^{2}}
$$

At small frequencies the main contribution to $G_{0}^{0}$ comes from the wavevectors close to the nodes, so we can do the summation over $\mathbf{k}$ and obtain:

$$
G_{0}^{0}\left(\omega_{n}\right) \approx \frac{i \omega_{n}}{\pi v^{2}} \ln \frac{\omega_{n}^{2}+\Lambda^{2}}{\omega_{n}^{2}},
$$

where $v=\sqrt{v_{F} v_{\Delta}}$ is the mean geometrical velocity near the nodes and $\Lambda$ denoting the upper cutoff of the order of $\Delta$. For the Hamiltonian (11) with $\mu=0, v_{F}=\varepsilon_{0} \sqrt{2}$, $v_{\Delta}=\Delta_{0} \sqrt{2}$.

From the equation (7) we see that the action (4) splits into two identical terms for the spin up and spin down polarizations so that:

$$
\begin{aligned}
S= & -N \operatorname{Tr} \ln \left(\frac{\partial}{\partial \tau}+\epsilon-\phi^{\dagger}(\tau) G_{\uparrow}\left(\frac{\partial}{\partial \tau}\right) \phi(\tau)\right) \\
& +\frac{N}{J} \int_{0}^{\beta} d \tau|\phi(\tau)|^{2}-\frac{N}{2} \epsilon \beta,
\end{aligned}
$$

where $N=2$ is the number of the spin channels,

$$
G_{\uparrow}\left(\frac{\partial}{\partial \tau}\right)=G_{0}^{0}\left(\frac{\partial}{\partial \tau}\right)\left(1+U G_{0}^{0}\left(\frac{\partial}{\partial \tau}\right)\right)^{-1} .
$$

Note that there is an additional term " $-N / 2 \epsilon \beta "$ in (10), which enforces the symmetry $\epsilon \rightarrow-\epsilon$ in the absence of the potential scattering. In 10 we find it more convenient to use a gauge, where $\phi$ is complex, but $\epsilon$ is time independent. In the large $N$ limit the action is given by the saddle point approximation [23]. So it is necessary to find the stationary point with respect to the auxiliary fields $\epsilon$ and $\phi$. Let us start with $\epsilon$ :

$$
\frac{2}{N} \frac{\partial S}{\partial \epsilon} \equiv I(\epsilon)=-\beta-\sum_{\omega_{n}} \frac{2}{\frac{\mathrm{e}^{-i \omega_{n} \delta}-1}{\delta}+\epsilon-G_{\uparrow}\left(\omega_{n}\right)|\phi|^{2}},
$$

where $\delta$ is an infinitesimal parameter showing the correct procedure of closing the Wick's contour. If $U=0$ then for any $\phi$ :

$$
I(\epsilon)=\beta\left(-1+2 g_{F}(\epsilon)\right) \quad \Longleftrightarrow \quad I(\epsilon)+I(-\epsilon)=0,
$$

where $g_{F}$ is the Fermi function. In fact this identity is just a consequence of the particle-hole symmetry. If $U \neq 0$ the relation above generalizes to

$$
I(\epsilon, U)+I(-\epsilon,-U)=0 .
$$

So in the absence of the potential scattering, $\epsilon=0$ at the saddle point. With help of (13), we write the saddle point condition as follows:

$$
\begin{aligned}
& \sum_{\omega_{n}} \frac{-\epsilon}{\left(i \omega_{n}-\epsilon+|\phi|^{2} G_{\uparrow}\left(\omega_{n}\right)\right)\left(i \omega_{n}+|\phi|^{2} G_{0}^{0}\left(\omega_{n}\right)\right)} \\
= & \sum_{\omega_{n}} \frac{U|\phi|^{2} G_{0}^{0}\left(\omega_{n}\right) G_{\uparrow}\left(\omega_{n}\right)}{\left(i \omega_{n}-\epsilon+|\phi|^{2} G_{\uparrow}\left(\omega_{n}\right)\right)\left(i \omega_{n}+|\phi|^{2} G_{0}^{0}\left(\omega_{n}\right)\right)},
\end{aligned}
$$

so that both sums become convergent at $\omega_{n} \rightarrow \infty$. Clearly $\epsilon=0$ as long as $|\phi|=0$. Near the phase transition the order parameter $|\phi|$ is small and therefore $\epsilon$ is also small. After introducing dimensionless parameters

$$
\epsilon \rightarrow \frac{\epsilon}{\Lambda}, \beta \rightarrow \Lambda \beta, U \rightarrow \frac{\Lambda U}{\pi v^{2}}, J \rightarrow \frac{\Lambda J}{\pi v^{2}},|\phi|^{2} \rightarrow \frac{|\phi|^{2}}{\pi v^{2}}
$$

and using $\epsilon \ll 1$ we can simplify (15) to:

$$
\tanh \frac{\epsilon \beta}{1+2|\phi|^{2} \ln \frac{1}{|\epsilon|}} \approx\left(2+4|\phi|^{2} \ln \frac{1}{|\epsilon|}\right) F(\phi, U),
$$

where

$$
F(\phi, U)=\int_{0}^{\infty} \frac{d x}{\pi} \frac{U|\phi|^{2}}{\left[\ln ^{-1}\left(1+\frac{1}{x^{2}}\right)+|\phi|^{2}\right]^{2}+U^{2} x^{2}} .
$$


The function $F$ increases linearly with $U$ at small $U$ and saturates at $1 / 2$ for large $U$. At finite temperature and small $U$ or $|\phi|$, (17) shows that $\epsilon \propto T$ up to logarithmic corrections, i.e. instead of (17) we can write:

$$
\tanh \frac{\varepsilon \beta}{1+2|\phi|^{2} \ln \beta} \approx 2\left(1+2|\phi|^{2} \ln \beta\right) F(\phi, U) .
$$

As long as $T$ is not too small we can ignore weak logarithmic dependence of the RHS of (19) on $T$, and $\epsilon$ is a decreasing function of temperature. This picture is valid down to

$$
T_{K}(\phi, U) \approx \exp \left(-\frac{1-2 F(\phi, U)}{4|\phi|^{2} F(\phi, U)}\right),
$$

at this temperature $\epsilon$ saturates becoming

$$
\epsilon_{0} \approx T_{K}\left(1+2|\phi|^{2} \ln \frac{1}{T_{K}}\right) .
$$

The quantity $T_{K}$ gives the low-energy scale of the problem and further will be identified with the Kondo temperature. Note that it has an exponential dependence on the order parameter $|\phi|$.

The second equation defining the saddle point is obtained differentiating (10) with respect to $\phi$. There is always a trivial solution $\phi=0$, the second one is found from:

$$
\frac{1}{J}=\int_{0}^{\infty} \frac{d \omega}{\pi} \Re\left\{\frac{G_{\uparrow}(\omega)}{i \omega-\epsilon+|\phi|^{2} G_{\uparrow}(\omega)}\right\},
$$

where the summation over discrete $\omega_{n}$ was transformed to the integration, which is valid in the low temperature limit. Assuming $\epsilon \ll 1$ and $\epsilon U \ll 1$, i.e. the system is in the vicinity of the phase transition, (22) becomes:

$$
\frac{1}{J}=\int_{0}^{\infty} \frac{d x}{\pi} \frac{\left(1+|\phi|^{2} \ln \left(1+\frac{1}{x^{2}}\right)\right) \ln \left(1+\frac{1}{x^{2}}\right)}{\left(1+|\phi|^{2} \ln \left(1+\frac{1}{x^{2}}\right)\right)^{2}+x^{2} U^{2} \ln ^{2}\left(1+\frac{1}{x^{2}}\right)}
$$

The RHS of 23) is bounded from above with the value 1 achieved at $\phi=0$ and $U=0$. Therefore we conclude that if $J$ is less than the critical value $J_{c}$ given by

$$
\frac{1}{J_{c}(U)}=\int_{0}^{\infty} \frac{d x}{\pi} \frac{\ln \left(1+\frac{1}{x^{2}}\right)}{1+x^{2} U^{2} \ln ^{2}\left(1+\frac{1}{x^{2}}\right)}
$$

then $\phi=0$ at the saddle point and the impurity is decoupled from quasiparticles. The critical coupling is an increasing function of $U$. In particular

$$
\begin{array}{ll}
J_{c}(U) \approx 1+\frac{1}{3} U^{2} & \text { at } U \ll 1, \\
J_{c}(U) \approx U & \text { at } U \gg 1 .
\end{array}
$$

As $J$ increases and becomes larger than $J_{c}$, the nontrivial solution of (23) becomes relevant, since it defines the minimum of the action as a function of $|\phi|^{2}$. Near the critical point $J \approx J_{c}$ we have:

$$
\begin{array}{ll}
|\phi|^{2} \approx \frac{J-J_{c}}{4 J_{c}^{2} \ln 2}\left(1+\frac{U^{2}}{2 \ln 2}\right) & \text { at } U \ll 1, \\
|\phi|^{2} \approx \frac{J-J_{c}(U)}{J_{c}(U)} & \text { at } U \gg 1,
\end{array}
$$

At the transition $T_{K}=0$ and according to (20) its asymptotics are:

$$
\begin{aligned}
& T_{K}(J, U) \approx \exp \left(-\frac{J_{c}^{2}(U) \ln 2}{2\left(J-J_{c}(U)\right)^{2} U}\right) \quad \text { at } U \ll 1, \\
& T_{K}(J, U) \approx \exp \left(-\frac{J_{c}^{2}(U)}{4\left(J-J_{c}(U)\right)^{2} \ln U}\right) \text { at } U \gg 1 .
\end{aligned}
$$

These expressions show that close to the phase transition $T_{K}$ is exponentially small and as long as $T_{K}<T \ll$ 1 , the system is in the regime where the effective impurity energy $\epsilon$ is a temperature dependent quantity (see (19)):

$$
\begin{aligned}
\epsilon & \approx \frac{1}{\beta}\left(1+\frac{J-J_{c}}{J_{c}^{2} 2 \ln 2} \ln \beta\right)^{2} \frac{\left(J-J_{c}\right) U}{J_{c}^{2}} \text { at } U \ll 1, \\
\epsilon & \approx \frac{1}{\beta}\left(1+2 \frac{J-J_{c}(U)}{J_{c}(U)} \ln \beta\right)^{2} \\
& \times \frac{2\left(J-J_{c}(U)\right) \ln U}{J_{c}(U)+2\left(J-J_{c}(U)\right) \ln U} \quad \text { at } U \gg 1 .
\end{aligned}
$$

The exponential behavior of the Kondo temperature and the effective impurity energy has been predicted already in 17. However, we note that this is the case only if the particle-hole symmetry is broken. In the particle-hole symmetric case $\epsilon$ and $T_{K}$ are identically equal to zero for the whole range of the magnetic coupling constant. In the regime, where $T \ll T_{K}, \epsilon$ saturates at the value proportional to $T_{K}(21)$.

As we noted in the introduction, our results are quite different from those derived in 16, 17]. We believe there are several weak points in those treatments. In particular, the action (2.28) in [16] clearly doesn't give the zero saddle point for the effective impurity energy $\epsilon_{f}$ at the particle-hole symmetric case $Q_{f}=N_{c} / 2$ (we use notations of 16] here). As a result the action in that form doesn't predict the Curie law for the susceptibility even when the impurity is free. On the other hand this symmetrization is appropriate for finding the order parameter $\phi$ and the critical coupling $g_{c}\left(J_{c}\right.$ in our notation). That is why we find completely different expressions for the Kondo temperature as compared to [16], while the critical coupling $J_{c}$ remains the same. In their second paper [17], the authors considered only the linear density of states (see (3.33) therein) and treated the action more carefully. However the saddle point equation (5.3) in [17] is still incorrect. It gives the nonzero value of $\epsilon_{f}$ in the particle-hole symmetric case (see e.g. (5.9) with $x=1 / 2$ ). It seems that there was lost a contribution to 


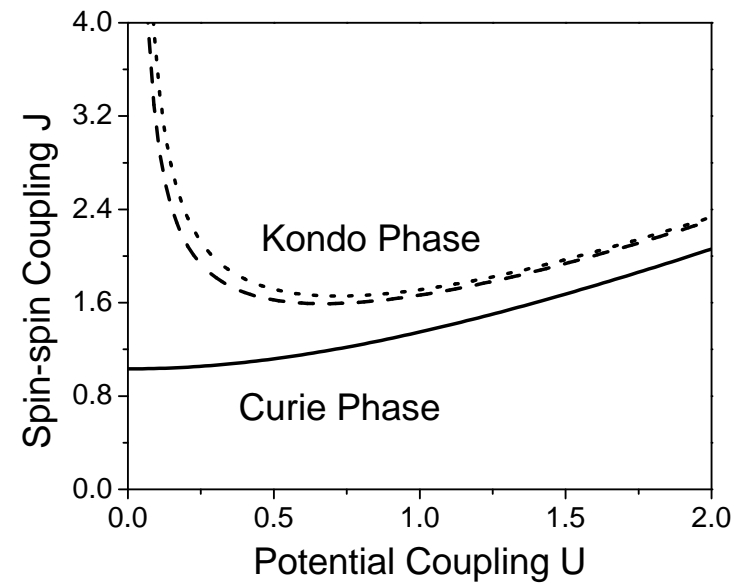

FIG. 1: Phase diagram in the $J-U$ plane. The solid line $(J=$ $J_{c}$ ) corresponds to the Kondo transition at zero temperature. At this coupling the order parameter $\phi$ first becomes non-zero. The dot and the dash line are the contours of constant Kondo temperature $T_{K}(J, U)=10^{-4}$ and $T_{K}=10^{-5}$ respectively. The critical spin-spin coupling $J_{c}$ remains finite at $U=0$, however in the particle-hole symmetric case there is no real transition to the Kondo phase since at any finite temperature the crossover value of $J$ (dash and dot lines) diverges as $U \rightarrow$ 0 . For $r<1$ the phase diagram is similar to this, while for $r>1$ the zero temperature boundary between the Kondo and Curie phases is analogous to the dash line. The solid line in this case doesn't represent any real phase transition.

(5.3) from the residue at $|\epsilon|>\left|\Delta_{0}\right|$, and hence an implicit particle-hole asymmetry was introduced.

Figure 1 illustrates the phase diagram in the $J-U$ plane. The solid line corresponds to the phase transition, while the dash and dot lines show the contours of the constant $T_{K}$. At fixed $T$ the latter lines separate hightemperature $\left(T_{K}<T\right)$ and the low-temperature $\left(T_{K}>\right.$ $T)$ regions. Here we would like to emphasize that the phase diagram with the dash line being a boundary is reminiscent to that obtained by NRG [20]. This probably indicates that the large $\mathrm{N}$-theory is not very reliable in the zero temperature limit. It is also important to note that the curve $J(U)$, corresponding to $T_{K}=$ const is very insensitive to the variations of the latter. Thus the dot and the dash lines corresponding to $T_{K}=10^{-4}$ and $T_{K}=$ $10^{-5}$ almost coincide.

\section{A. Generalization to the nonlinear density of states}

Before we proceed further with D-wave superconductors, let us generalize obtained in the large $\mathrm{N}$ limit expressions to the case of vanishing density of states with an arbitrary exponent:

$$
\rho(\epsilon)=\left\{\begin{array}{cc}
\frac{r+1}{2}|\epsilon|^{r} & |\epsilon|<1 \\
0 & |\epsilon| \geq 1
\end{array}\right.
$$

I.e. the action is still given by the equation (10) but the unperturbed Green function $G_{0}^{0}\left(\omega_{n}\right)$ is now as follows:

$G_{0}^{0}\left(\omega_{n}\right)=i \int_{-1}^{1} \frac{\omega_{n} \rho(\epsilon)}{\omega_{n}^{2}+\epsilon^{2}} d \epsilon=i \frac{r+1}{2} \frac{\mathcal{F}\left(1, \frac{1+r}{2}, \frac{3+r}{2},-\frac{1}{\omega_{n}^{2}}\right)}{\omega_{n}}$,

where $\mathcal{F}$ is the hypergeometric function. High and low frequency asymptotics of $G_{0}^{0}$ are

$$
G_{0}^{0}\left(\omega_{n}\right) \approx\left\{\begin{array}{cc}
i \omega_{n}^{r} \frac{r+1}{2} \frac{\pi}{\cos \frac{\pi r}{2}}-i \omega_{n} \frac{r+1}{r-1} & \omega_{n} \ll 1 \\
i \omega_{n}^{-1} & \omega_{n} \gg 1 .
\end{array}\right.
$$

For $r<1 / 2$ the problem was studied in detail in [14, 15. Both the consequent analysis and numerical results [20] show that the effects of the particle-hole asymmetry become crucial for $r>1 / 2$. Here only this situation will be considered. Doing the same steps as before we find the critical $J$, where $|\phi|$ first becomes nonzero, is

$$
\begin{array}{lll}
J_{c} \sim \frac{r(r+1)}{2} & \text { at } U \rightarrow 0 \\
J_{c} \sim U \max \left(\frac{2 r}{r+1}, 1\right) & \text { at } U \rightarrow \infty .
\end{array}
$$

In the case of the small potential scattering $U$, the order parameter near the phase transition is:

$$
|\phi|^{2} \sim \frac{J-J_{c}}{J_{c}^{2}} \frac{4 r-2}{(r+1)^{2}}\left(\psi\left(\frac{r+1}{2}\right)-\psi\left(\frac{1}{2}\right)\right)^{-1},
$$

where $\psi$ is the digamma function. Note that regardless of $r,|\phi|^{2}$ is proportional to $J-J_{c}$. However, when $\epsilon$ is considered, it is necessary to distinguish between $r<1$ and $r>1$. In the former case one can define the Kondo temperature:

$$
T_{K} \approx\left[\frac{2 \cos \frac{\pi r}{2}}{\pi^{r}(1-r)}+\frac{4^{r}-1}{4^{r-1}} \frac{\cos \frac{\pi r}{2} \zeta(2 r)}{\pi^{1+r}|\phi|^{4} F_{r}(U)}\right]^{-\frac{1}{1-r}},
$$

where $\zeta$ is the Riemann zeta function,

$$
\begin{array}{ll}
F_{r}(U) \sim \frac{U}{2} \frac{(r+1)^{2}}{2 r-1}\left(\psi\left(\frac{1+r}{2}\right)-\psi\left(\frac{r}{2}\right)\right) & \text { for } U \ll 1 \\
F_{r}(U) \sim \frac{U^{\frac{1-r}{r}}-1}{\left(\cos \frac{\pi r}{2}\right)^{\frac{1}{r}}} \frac{\pi^{\frac{1}{r}}(1+r)^{\frac{1}{r}}}{r 2^{\frac{1+r}{r}} \sin \frac{\pi}{2 r}} & \text { for } U \gg 1 .
\end{array}
$$

For $r<1$ near the phase transition we have:

$$
T_{K} \propto\left(J-J_{c}\right)^{\frac{2}{1-r}}
$$

Above the Kondo temperature $\epsilon$ increases with $T$, while for $T<T_{K}$ it saturates at the value:

$$
\epsilon \sim|\phi|^{2} \frac{\pi^{1+r}(1+r)}{2 \cos \frac{\pi r}{2}} T_{K}^{r}\left(1-\frac{2 \cos \frac{\pi r}{2}}{\pi^{r}(1+r)} T_{K}^{1-r}\right) .
$$


It is easy to check that the expressions for $r=1$ obtained earlier are consistent with these formulas.

The picture becomes quite different for $r>1$. If the order parameter is sufficiently small, $\epsilon$ can be found from:

$$
\tanh \left(\frac{\beta \epsilon}{1+\frac{r+1}{r-1}|\phi|^{2}}\right)=2|\phi|^{2}\left(1+\frac{r+1}{r-1}|\phi|^{2}\right) F_{r}(U) .
$$

Provided the RHS of (38) is less than 1, this equation has a solution with $\epsilon \propto T$, i.e. vanishing as $T$ goes to zero. On the other hand if the RHS becomes greater than 1, this equation has no solution and at zero temperature at this point there is a phase transition to the Kondo phase. Thus for $r$ slightly greater than 1 and $U \ll 1$ the new critical coupling $\tilde{J}_{c}$ is

$$
\tilde{J}_{c} \sim J_{c}+0.35 \sqrt{\frac{r-1}{U}} .
$$

For $U \gg 1$ we have $\tilde{J}_{c} \approx J_{c}$. The diverging behavior of $\tilde{J}_{c}$ at $U \rightarrow 0$ is in fact very similar to that of the crossover spin-spin coupling for $r=1$ at finite temperature (see dash line on fig. 1). However, for $r>1$ this divergence exists even in the zero temperature limit.

At this point we get another evidence that the large$\mathrm{N}$ theory might be not reliable at small temperatures for $r \leq 1$. Namely, the NRG analysis shows that $r=1$ is not a special point in the phase diagram [21]. On the other hand, the saddle point results predict that the curve similar to the dash (not solid) line in figure 1 represents the actual transition to the Kondo phase for $r>1$. At the same time at finite temperature the mean field phase diagram is qualitatively similar to the NRG result [15, 21. And moreover, for $r$ close to 1 the contours $T_{K}(J, U)=T$ are very insensitive to the value of $T$ (see figure 1 ).

\section{MAGNETIC SUSCEPTIBILITY AND QUASIPARTICLE DENSITY OF STATES.}

We start this section from calculating a local magnetic susceptibility at zero magnetic field. In the saddle point approximation it is given by:

$$
\chi_{l o c}=-\frac{1}{4 \beta} \frac{\partial^{2} S_{e f f}(h)}{\partial h^{2}}=-\frac{1}{4 \beta} \frac{\partial^{2} S_{e f f}(\epsilon)}{\partial \epsilon^{2}} .
$$

Using (12) we immediately get:

$$
\chi_{l o c}=\sum_{\omega_{n}} \frac{N}{4 \beta\left(\omega_{n}-i \epsilon-i|\phi|^{2} G_{\uparrow}\left(\omega_{n}\right)\right)^{2}} .
$$

This expression becomes particularly simple if there is no potential scattering and $\epsilon=0$ (we always assume that the temperature is small compared to the cutoff, i.e. $\beta \gg 1$ ):

$$
\chi_{l o c} \approx \frac{N \beta}{16}\left(1+2|\phi|^{2} \log \frac{\beta}{\pi}\right)^{-2} .
$$
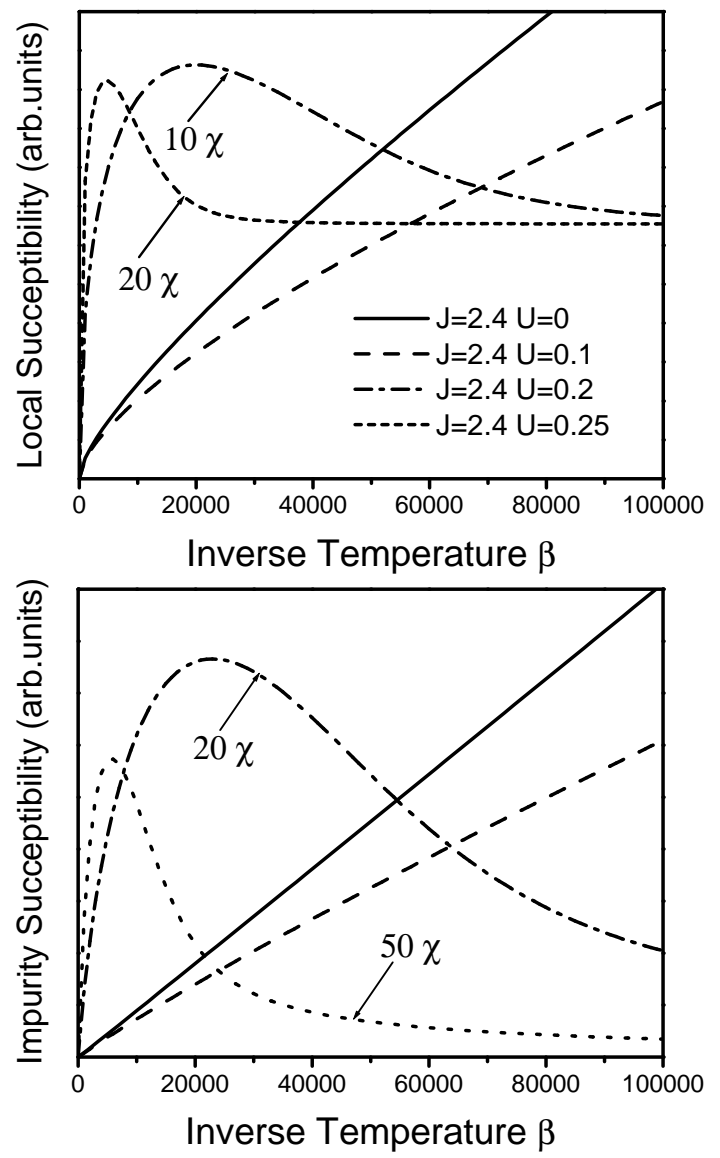

FIG. 2: Temperature dependence of the local susceptibility (top) and the impurity susceptibility (bottom) in the Kondo phase for different potential couplings. For $U=0$ and $U=0.1$ (see Figure 1) the Kondo temperature is much smaller than $T=\beta^{-1}$ for all plotted values of $\beta$. So both susceptibilities increase with $\beta$. Note that $\chi_{l o c}$ is clearly a sublinear function of inverse temperature even in the particle-hole symmetric case. For $U=0.2$ and $U=0.25$ the Kondo temperature becomes large enough and both susceptibilities have a maximum at $\beta T_{K} \approx 1$. The local susceptibility saturates at $\beta \rightarrow \infty$, while $\chi_{i m p} \rightarrow 0$ (see Eq. 52).

It is not surprising that for $\phi=0$ in the physical case $N=$ 2, (42) gives one half of the usual Curie constant. This feature is an artifact of the large $\mathrm{N}$ techniques applied here. For $r<1$ instead of (42) we have

$$
\begin{aligned}
& \chi_{l o c} \approx \frac{N \beta}{16} \quad 1 \ll \beta \ll \beta_{0} \equiv \pi\left[\frac{2 \cos \frac{\pi r}{2}}{\pi(r+1) \phi^{2}}\right]^{\frac{1}{1-r}} \\
& \chi_{l o c} \approx \frac{2 N \beta^{2 r-1} \cos ^{2} \frac{\pi r}{2}}{\phi^{4} \pi^{2(r+1)}(r+1)^{2}} \frac{4^{r}-1}{4^{r}} \zeta(2 r) \beta \gg \beta_{0} .
\end{aligned}
$$

On the other hand for $r>1$ the local susceptibility in the particle-hole symmetric case is completely described by the Curie law.

The formulas above are valid as long as $\epsilon \ll T$. For $r \leq 1$ this is equivalent to $T \gg T_{K}$. Below the Kondo 
temperature $\chi_{l o c}$ saturates at:

$$
\begin{array}{ll}
\chi_{l o c} \approx \frac{N|\phi|^{2}}{4 T_{K}\left(1-2|\phi|^{2} \ln \left(T_{K}\right)\right)^{3}} & \text { for } r=1, \\
\chi_{l o c} \approx \frac{N}{4|\phi|^{4} T_{K}^{2 r-1}} \frac{2(1-r) \cos ^{2} \frac{\pi r}{2}}{r^{2} \pi^{2 r-1}(r+1)^{2}} & \text { for } r<1 .
\end{array}
$$

The temperature dependence of $\chi_{l o c}$ for $r=1$ is plotted on the top graph on Figure 2. This function is nonmonotonic with the maximum occurring at $T \approx T_{K}$.

Using equation (41), it is not hard to calculate a correction to $\chi_{l o c}(T=0)$ for $T \ll T_{K}$ :

$$
\chi_{l o c}(T)-\chi_{l o c}(0) \approx \frac{9 \zeta(3)|\phi|^{2} T^{3}}{\pi \epsilon^{4}}\left(1+2|\phi|^{2} \ln \frac{1}{T}\right),
$$

showing that $\chi_{l o c}$ decreases at $T \rightarrow 0$ for $T<T_{K}$.

The local susceptibility is the response function to the magnetic field coupled only to the impurity. We will also consider here the impurity susceptibility which additionally includes the contribution from the nearby electron cloud .

Assuming that g-factors of band and impurity electrons are the same we see that the only effect of the magnetic field $h$ on the action (10) is the shift of the imaginary frequency $\omega_{n} \rightarrow \omega_{n}+i h$ for the spin up states and $\omega_{n} \rightarrow \omega_{n}-i h$ for the spin down states. Therefore, the impurity susceptibility at zero field is:

$$
\begin{aligned}
\chi_{i m p} & =\frac{N}{4 \beta} \sum_{n} \frac{\left(1-i|\phi|^{2} \frac{\partial}{\partial \omega_{n}} G_{\uparrow}\left(\omega_{n}\right)\right)^{2}}{\left(\omega_{n}+i \epsilon-i|\phi|^{2} G_{\uparrow}\left(\omega_{n}\right)\right)^{2}} \\
& -\frac{N}{4 \beta} \sum_{n} \frac{|\phi|^{2} \frac{\partial^{2}}{\partial \omega_{n}^{2}} G_{\uparrow}\left(\omega_{n}\right)}{i \omega_{n}-\epsilon+|\phi|^{2} G_{\uparrow}\left(\omega_{n}\right)} .
\end{aligned}
$$

If $\epsilon=0$, expression above reduces to:

$$
\chi_{i m p} \approx \frac{N \beta}{16}\left(1-\frac{2|\phi|^{2}}{1+2|\phi|^{2} \ln \beta}\right) .
$$

Note that $\chi_{i m p}$ asymptotically approaches Curie law at $T \rightarrow 0$, contrary to 42 where $T \chi_{l o c} \rightarrow 0$. This peculiarity was already predicted in 24], however in the later work [20], $\chi_{l o c}$ was found to be proportional to the inverse temperature without any logarithmic corrections. The controversy between the mean-field and NRG results can not be resolved considering the next terms in the $1 / N$ expansion near the saddle point. In fact, it is easy to show, that for $U=0$ at each order of the perturbation series for $\chi_{l o c}$, a multiplier $\left(1+2|\phi|^{2} \ln \beta\right)^{2}$ is generated in the denominator. Therefore the sublinear dependence of $\chi_{l o c}(T)$ is generic for the large $N$ approach. Probably the reason for the discrepancy between this work and 20, 21] is that the large $N$ expansion is asymptotic in the particle-hole symmetric case and for $r<1$, and it diverges as temperature goes to zero.

For $r>1$ near $J=J_{c}, \chi_{i m p}=\chi_{l o c}=N \beta / 16$. While for $r<1$ there is a crossover between

$$
\chi_{i m p} \approx \frac{N \beta}{16}
$$

and

$$
\chi_{i m p} \approx \frac{N r \beta}{16},
$$

$T \beta_{0} \gg 1$ and $T \beta_{0} \ll 1$ respectively, $\beta_{0}$ is defined in (43). So the impurity is partially screened. We note again that these results are valid only for $r>1 / 2$, otherwise there is a transition to Kondo phase even in the particle-hole symmetric case.

Below the Kondo temperature $\chi_{i m p}$ goes to zero in agrement with [17]. For example at $r=1$ :

$$
\chi_{i m p} \approx \frac{T|\phi|^{2} N \ln 2}{T_{K}^{2}\left(1-2|\phi|^{2} \ln T\right)} .
$$

Impurity susceptibility as a function of temperature is plotted on the bottom graph on Figure 2.

Another manifestation of the coupling between quasiparticles and impurity is the change of the electron density of states, which is given by the imaginary part of the effective Green function:

$$
\rho(\Omega, \mathbf{r})=\Im \operatorname{Tr} \frac{1+\tau_{z}}{2} \tilde{G}(\mathbf{r}, \mathbf{r}, \Omega),
$$

where $\Omega$ is the real frequency with an infinitesimal positive imaginary part,

$$
\tilde{G}\left(\mathbf{r}, \mathbf{r}^{\prime}, \Omega\right)=G\left(\mathbf{r}, \mathbf{r}^{\prime}, \Omega\right)+G\left(\mathbf{r}, \mathbf{r}_{0}, \Omega\right) \mathcal{T}_{K}(\Omega) G\left(\mathbf{r}_{0}, \mathbf{r}^{\prime}, \Omega\right) .
$$

The scattering matrix $\mathcal{T}_{K}(\Omega)$, corresponding to the Kondo contribution is equal to:

$$
\mathcal{T}_{K}(\Omega)=\frac{|\phi|^{2}}{-\Omega+\epsilon \tau_{z}-|\phi|^{2} \tau_{z} G\left(\mathbf{r}_{0}, \mathbf{r}_{0}, \Omega\right) \tau_{z}},
$$

note that a similar form for $\mathcal{T}$ was found in [19] for the case of an Anderson impurity below the Kondo temperature. Using (7), (53) can be simplified further:

$$
\rho(\Omega, \mathbf{r})=\Im G_{+}^{0}(\mathbf{r}, \Omega) \mathcal{T}_{+}(\Omega) G_{+}^{0}(-\mathbf{r}, \Omega)+G_{x}^{0}(\mathbf{r}, \Omega) \mathcal{T}_{-}(\Omega) G_{x}^{0}(-\mathbf{r}, \Omega) .
$$


We adopted the notation:

$$
\begin{gathered}
G_{+}^{0}(\mathbf{r}, \Omega)=\operatorname{Tr} \frac{1+\tau_{z}}{2} G_{0}(\mathbf{r}, \Omega), \quad G_{x}^{0}(\mathbf{r}, \Omega)=\operatorname{Tr} \frac{\tau_{x}}{2} G_{0}(\mathbf{r}, \Omega), \\
\mathcal{T}_{ \pm}(\Omega)=-\frac{1}{1 \pm U G_{0}^{0}(\Omega)}+\left(\frac{1}{1 \pm U G_{0}^{0}(\Omega)}\right)^{2} \frac{1}{-\Omega \pm \epsilon-|\phi|^{2} \frac{G_{0}^{0}(\Omega)}{1 \pm U G_{0}^{0}(\Omega)}} .
\end{gathered}
$$

The spatial dependence of DOS coincides with that obtained earlier for the case of the pure classical scattering [10]. We note that at small frequencies $(\Omega \ll \Delta)$, there is a maximum at the nearest neighbors of the impurity and at $r \rightarrow \infty$ the asymptotic behavior of the DOS is $r^{-2}$. Also it is easy to show that if the resonance frequency is much smaller than the superconducting gap, the spatially integrated DOS remains particle-hole symmetric. It is determined by the momenta in the vicinity of the nodes and therefore this result is valid regardless the microscopical details of the model such as chemical potential, existence of second-neighbor hopping matrix elements, etc.

Clearly the $\mathcal{T}$-matrix for the Kondo scattering channel has a pole at the frequency $\Omega \approx T_{K}$. If $T_{K} \ll \Delta$, i.e. $J \approx J_{c}$, the resonant levels corresponding to the poles in $\mathcal{T}_{+}$and $\mathcal{T}_{-}$become very narrow and they have roughly Lorentzian shape. In the opposite limit $\Delta \ll T_{K}$, the resonance corresponding to $\mathcal{T}_{-}$disappears and (56) results in the Fano lineshape in agreement with [26].

We would like to emphasize that the scattering on a strong classical magnet gives two identical peaks at posi- tive and negative frequencies and additional weak potential coupling results in the weak splitting of those [10]. In the Kondo case, even at small $U$ the spatial structure of the resonances at positive and negative frequency is highly asymmetric.

\section{NON-MAGNETIC IMPURITY IN A D-WAVE SUPERCONDUCTOR}

In this section we focus on the situation, when the spin is not located on the impurity site, but rather it is a staggered moment distributed nearby. The main motivation to this investigation comes from the NMR experiments 4, 5, 6, 7, 8, 9, with non-magnetic $\mathrm{Zn}$ or $\mathrm{Li}$ impurities in a BSSCO. The effective strength of the induced moments rapidly decreases with the distance [9, therefore it is reasonable to assume that the spins are sitting only on the first nearest neighbors.

In this way the new interacting part of the Hamiltonian becomes:

$$
H_{\text {int }}=U \psi_{i}^{\dagger}\left(\mathbf{r}_{0}\right) \tau_{z}^{i j} \psi_{j}\left(\mathbf{r}_{0}\right)-\frac{J}{2} \sum_{s \in O}\left(\psi_{\uparrow}^{\dagger}\left(\mathbf{r}_{s}\right) F_{\uparrow}-F_{\downarrow}^{\dagger} \psi_{\downarrow}\left(\mathbf{r}_{s}\right)\right)\left(F_{\uparrow}^{\dagger} \psi_{\uparrow}\left(\mathbf{r}_{s}\right)-\psi_{\downarrow}^{\dagger}\left(\mathbf{r}_{s}\right) F_{\downarrow}\right),
$$

where $O$ denotes the subset of the sites closest to the $\mathbf{r}_{0}$. Contrary to the spin-spin coupling which is strongest on the nearest neighbors of the impurity, the potential scattering is dominated by the local term, therefore its form is unchanged as compared to (2). Repeating the same steps as before and using the static approximation we obtain:

$$
S=-\operatorname{Tr} \ln \left(\frac{\partial}{\partial \tau}+\epsilon \tau_{z}-\sum_{s, s^{\prime} \in O} \phi_{s} G\left(\mathbf{r}_{s}, \mathbf{r}_{s^{\prime}}, \frac{\partial}{\partial \tau}\right) \phi_{s^{\prime}}\right)+\frac{2 \beta}{J} \sum_{s \in O} \phi_{s}^{2},
$$

This action can be also evaluated in the saddle point approximation. The important difference with the local spin case is that there are four nontrivial saddle points corresponding to $S\left(\phi_{10}=\phi_{01}=\phi_{\overline{1}, 0}=\phi_{0, \overline{1}}\right)$, $D\left(\phi_{10}=-\phi_{01}=\phi_{\overline{1}, 0}=-\phi_{0, \overline{1}}\right)$, and $P\left(\phi_{10}=-\phi_{\overline{1}, 0} ;\right.$ $\left.\phi_{0,1}=\phi_{0, \overline{1}}=0\right)$ symmetry of the order parameter, the latter case being twice degenerate. On the Figure 3 we show the dependence of the critical coupling $J_{c}$ on the potential scattering for the order parameter of the $S$ and $D$ symmetry ( $P$ case is always in between) and for the local spin. Clearly, for the whole range of $U, D$ order has a lower $J_{c}$ and as a result a smaller action. Also, contrary to the local spin case, $J_{c}$ decreases with $U$. This phenomenon is readily understood since the potential scattering increases the quasiparticle density of states on the nearest neighbors [10], where the magnetic moments are 


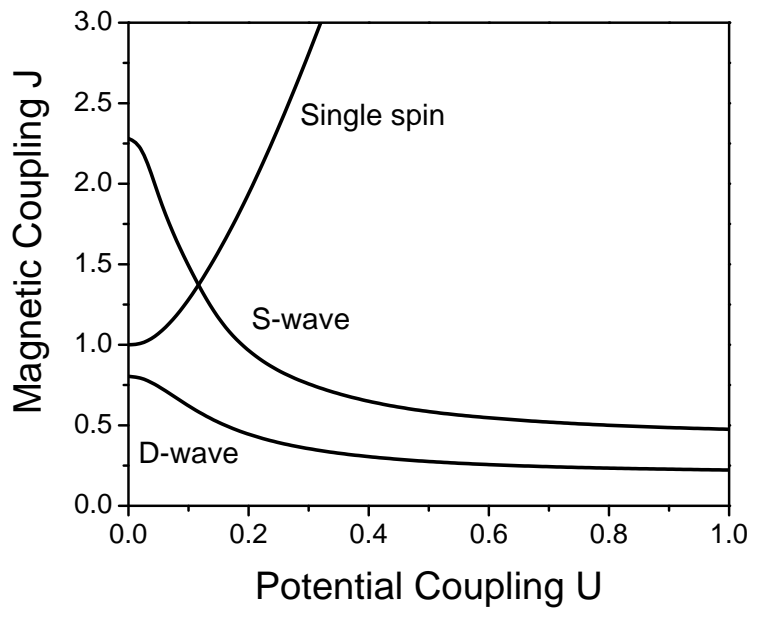

FIG. 3: Critical spin-spin coupling as a function of potential scattering for the non-local spin case. The D-wave configuration of the order parameter $\phi$ always has a lower critical coupling $J_{c}$ and the smallest action. Contrary to the local spin case the Kondo transition occurs at lower value of $J$ with increasing $U$.

located, and hence enhances the effective spin-spin coupling. It is interesting to note, that the effective DOS of the "bath" electrons for the $D$-wave saddle point in the absence of potential scattering term is proportional to $\omega^{3}$ and not $\omega$ due to Green functions cancellation near the nodes [27. However this feature is not important for our consequent analysis.

Both local and impurity magnetic susceptibility practically don't change as compared to the local spin case, since they are sensitive to the value of the impurity spin and not to its distribution. The situation is quite different for the density of states. In particular, for the spin scattering part and $D$-wave order parameter (56) generalizes to

$$
\begin{aligned}
\rho(\Omega, \mathbf{r}) & =\Im G_{+}^{d}(\mathbf{r}, \Omega) \mathcal{T}_{+}(\Omega) G_{+}^{d}(-\mathbf{r}, \Omega) \\
& +G_{x}^{d}(\mathbf{r}, \Omega) \mathcal{T}_{-}(\Omega) G_{x}^{d}(-\mathbf{r}, \Omega)
\end{aligned}
$$

where:

$$
\begin{gathered}
\mathcal{T}_{ \pm}(\Omega)=\frac{-1}{\Omega \mp \epsilon+|\phi|^{2}\left(G_{0}^{d}(\Omega)+\frac{G_{x}^{d^{2}}\left(\mathbf{r}_{0}, \Omega\right) U^{2} G_{0}^{0}(\Omega)}{1-U^{2} G_{0}^{02}(\Omega)}\right)}, \\
G_{+, x}^{d}(\mathbf{r}, \Omega)=\sum_{s \in O}\left(s_{x}^{2}-s_{y}^{2}\right) G_{+, x}\left(\mathbf{r}, \mathbf{r}_{s}, \Omega\right), \\
G_{0}^{d}(\Omega)=\sum_{s, s^{\prime} \in O}\left(s_{x}^{2}-s_{y}^{2}\right)\left(s_{x}^{\prime 2}-s_{y}^{\prime 2}\right) G_{0}^{0}\left(\mathbf{r}_{s}-\mathbf{r}_{s^{\prime}}, \Omega\right),
\end{gathered}
$$

with $s_{i}=0, \pm 1$. The resonance frequency is approximately equal to the Kondo temperature as in the local spin case. If the potential scattering is not too large $U G_{0}^{0}\left(T_{K}\right) \ll 1$ then the spatial dependence of the DOS is determined by the interference from the scattering on the four nearest neighbors to the impurity sites. In particular, for the $D(S)$ order parameter, the resonance corresponding to $\mathcal{T}_{-}\left(\mathcal{T}_{+}\right)$matrix gives the largest DOS on the impurity site $\mathbf{r}=\mathbf{r}_{0}$, local maximum on the second neighbors and very small signal on the first neighbors [13]. This behavior is in the excellent agreement with the experiments, where the spatial distribution of DOS near the Zn impurity was directly observed [3]. Also at $r \rightarrow \infty$ the density of states vanishes as $r^{-4}$, i.e. much faster than for the the local spin case. The spatially integrated DOS is generically particle-hole asymmetric. In particular it can be shown that if $U G_{0}^{0}\left(T_{K}\right) \ll 1$ and $T_{K} \ll \Delta$ then at the resonance frequency the integrated DOS is:

$$
\begin{gathered}
\rho_{+}(\Omega) \approx \frac{4}{\Delta_{0}^{2}} \int \frac{\varepsilon_{k}^{2} \Delta_{k}^{2}}{\left(\varepsilon_{k}^{2}+\Delta_{k}^{2}\right)^{2}} \frac{d^{2} k}{(2 \pi)^{2}} \Im \mathcal{T}_{+}(\Omega) \\
\rho_{-}(-\Omega) \approx \frac{4}{\Delta_{0}^{2}} \int \frac{\Delta_{k}^{4}}{\left(\varepsilon_{k}^{2}+\Delta_{k}^{2}\right)^{2}} \frac{d^{2} k}{(2 \pi)^{2}} \Im \mathcal{T}_{-}(-\Omega)
\end{gathered}
$$

Simple power counting shows that (63) and (64) converge near the nodes. Therefore the ratio of the spatially integrated DOS is nonuniversal and while for particle-hole symmetric spectrum $\rho_{-} / \rho_{+} \sim 2.5$, it decreases with the doping crossing 1 at some moment. For comparison we will give the results for the local spin:

$$
\begin{gathered}
\tilde{\rho}_{+}(\Omega) \approx \int_{\varepsilon_{k}^{2}+\Delta_{k}^{2}>\Omega^{2}} \frac{\varepsilon_{k}^{2}}{\left(\varepsilon_{k}^{2}+\Delta_{k}^{2}\right)^{2}} \frac{d^{2} k}{(2 \pi)^{2}} \Im \mathcal{T}_{+}(\Omega) \\
\tilde{\rho}_{-}(-\Omega) \\
\approx \int_{\varepsilon_{k}^{2}+\Delta_{k}^{2}>\Omega^{2}} \frac{\Delta_{k}^{2}}{\left(\varepsilon_{k}^{2}+\Delta_{k}^{2}\right)^{2}} \frac{d^{2} k}{(2 \pi)^{2}} \Im \mathcal{T}_{-}(-\Omega)
\end{gathered}
$$

This expressions logarithmically diverge when $\varepsilon$ and $\Delta$ become small, therefore it is necessary to put a low energy cutoff of the order of frequency. The integrals mainly sit on the nodes and $\rho_{+} \approx \rho_{-}$if $\Omega$ is small. Physically this differences are related to the slow $\left(1 / r^{2}\right)$ dependence of the DOS on the distance for a single spin, while for the nonlocal scattering the DOS rapidly decreases away from the impurity and the main contribution to the spatial integrals comes from the short distances or high momenta.

Let us say a few words about strong potential scattering case $U \gg 1$. As we see from the Figure 3 , the critical Kondo coupling $J_{c}$ goes to zero, in fact as $1 / U$ up to $\log$ arithmic corrections. Therefore for the most values of $J$ the system is in the Kondo phase. If $J$ is not too large, then the order parameter $|\phi|$ and the Kondo temperature are small enough so that the condition $U G_{0}^{0}\left(T_{K}\right) \ll 1$ fulfills. As a result the spatial structure of the DOS remains the same as if there is no potential scattering. This contradicts the naive idea of the "hard-wall" impurity, which prohibits large DOS at $\mathbf{r}=\mathbf{r}_{0}$. And the reason, why this argument is not correct is that the energy scale $T_{K}$ is so small that the potential scattering is irrelevant. If both conditions $U \gg 1$ and $J \gg J_{c}$ are fulfilled then $U G_{0}^{0}\left(T_{K}\right)$ might be greater than 1 . In this case the Kondo scattering occurs on the almost frozen density of states created 
by the potential scatterer and apart from small variations the spatial structures of the Kondo and potential resonances coincide. But we emphasize again that in order to have this situation both $U$ and $J$ should be very large.

\section{CONCLUSIONS}

We have studied the screening of a magnetic impurity in $D$-wave superconductors. It is found that there is a Kondo transition, which occurs only if the particle-hole symmetry is distorted, e.g. by the potential scattering. The Kondo temperature is exponentially small near the critical coupling in agreement with [17. However, at finite but small temperature the crossover from the regime $T \gg T_{K}$, where the impurity spin is not screened to $T \ll T_{K}$ occurs at qualitatively different values of the magnetic coupling $J$ (see (fig. 1)), in particular $J \rightarrow \infty$ if the potential coupling $U \rightarrow 0$. In the particle-hole symmetric phase it was found that the impurity susceptibility behaves in a Curie-like fashion: $T \chi_{i m p} \rightarrow$ const as $T \rightarrow$ zero, while the local susceptibility has multiplicative logarithmic corrections, so that: $T \chi_{l o c} \rightarrow 0$. This result, which holds in any order of $1 / N$ expansion, agrees with 24], however it contradicts later NRG investigations 20, 21]. It is possible that the logarithmic corrections are beyond the accuracy of the large $N$ expansion in the particle-hole symmetric case. On the other hand, the resulting phase diagram at finite temperature is qualitatively similar to that, obtained by NRG [20, 21] and dynamic multichannel [22] methods. Moreover the contours of constant $T_{K}$ change very slowly (logarithmically) with $T_{K}$ (see fig. 1 ). Therefore, we expect that the conclusions about the Kondo phase are reliable.

Also we examined the situations with $1 / 2<r<1$ and $r>1$. The former appears to be analogous to the case $r=1$ with the only difference that exponential (logarithmical) behavior is substituted by the suitable power law. For $r>1$ the picture is different in a sense that the phase diagram is qualitatively consistent with NRG results even in the zero temperature limit.

Finally we studied the behavior of a non-magnetic impurity in a D-wave superconductor, which creates a staggered magnetization on the nearest copper neighbors. It was found that the saddle point corresponding to the true minimum of the action always has a $D$-symmetry. The resulting spatial structure of the quasiparticle density of states near the impurity for this saddle point is consistent with experimental data. It was also shown that unlike the single spin case the critical magnetic coupling corresponding to the Kondo transition decreases as $U$ increases .

\section{Acknowledgments}

The author is grateful to W.A. Atkinson and H. Alloul for useful discussions and especially to S. Sachdev and M. Vojta for many very important comments and suggestions.
[1] E.W. Hudson, S.H. Pan, A.K. Gupta, K.W. Ng, J.C. Davis, Science 285, 88 (1999).

[2] E.W. Hudson, K.M. Lang, V. Madhavan, S.H. Pan, H. Eisaki, S. Uchida, J.C. Davis, cond-mat/0104237.

[3] S.H. Pan, E.W. Hudson, K.M. Lang, H. Eisaki, S. Uchida, J.C. Davis, Nature 403, 746 (2000).

[4] J. Bobroff, W. A. MacFarlane, H. Alloul, P. Mendels, N. Blanchard, G. Collin, and J.-F. Marucco , Phys. Rev. Lett. 83, 4381 (1999).

[5] W.A. MacFarlane, J. Bobroff, H. Alloul, P. Mendels, N. Blanchard, G. Collin, J.-F. Marucco, cond-mat/9912165.

[6] A.V. Mahajan, H. Alloul, G. Collin, J. F.Marucco, Eur. Phys. J B 13, 457 (2000).

[7] J. Bobroff, H. Alloul, W.A. MacFarlane, P. Mendels, N. Blanchard, G. Collin, J.-F. Marucco, cond-mat/0010234.

[8] P. Mendels, J. Bobroff, G. Collin, H. Alloul, M. Gabay, J.F. Marucco, N. Blanchard, B. Grenier, Europhys. Lett. 46, 678 (1999).

[9] M.-H. Julien, T. Fehér, M. Horvatic, C. Berthier, O. N. Bakharev, P. Ségransan, G. Collin, and J.-F. Marucco , Phys. Rev. Lett. 84, 3422 (2000).

[10] A.V. Balatsky, M.I. Salkola, and A. Rosengren, Phys. Rev. B 51, 15547 (1995), M.I. Salkola, A.V. Balatsky, and D.J. Scalapino, Phys. Rev. Lett. 77, 1841 (1996), I. Martin, A.V. Balatsky, J. Zaanen, cond-mat/0012446.

[11] M.E. Flatté, Phys. Rev. B 61, R14920 (2000).

[12] W.A. Atkinson, P. J. Hirschfeld, A. H. MacDonald, and
K. Ziegler, Phys. Rev. Lett. 85, 3926 (2000).

[13] Anatoli Polkovnikov, Subir Sachdev, and Matthias Vojta, Phys. Rev. Lett. 86, 296 (2001).

[14] David Withoff and Eduardo Fradkin, Phys. Rev. Lett. 64, 1835 (1990).

[15] Kevin Ingersent and Qimiao Si, cond-mat/9810226.

[16] Carlos R. Cassanello and Eduardo Fradkin, Phys. Rev. B 53, 15079 (1996).

[17] Carlos R. Cassanello and Eduardo Fradkin, Phys. Rev. B 56, 11246 (1997).

[18] Jian-Xin Zhu and C.S. Ting, Phys. Rev. B 63, 020506(R) (2001).

[19] Guang-Ming Zhang, Hui Hu, and Lu Yu, Phys. Rev. Lett. 86, 704 (2001).

[20] Carlos Gonzalez-Buxton and Kevin Ingersent, Phys. Rev. B 57, 14254 (1998).

[21] M. Vojta and R. Bulla, in preparation.

[22] M. Vojta, cond-mat/0102089.

[23] N. Read and D.M. Newns, J. Phys. C 16, 3272 (1983).

[24] K. Chen, C. Jayaprakash, J. Phys.: Condensed Matter 7, L491 (1995).

[25] A.C. Hewson, The Kondo Problem to Heavy Fermions, Cambridge University Press, Cambridge (1997).

[26] O. Újsághy, J. Kroha, L. Szunyogh, and A. Zawadowski, Phys. Rev. Lett. 85, 2557 (2000).

[27] The author is grateful to M. Vojta for pointing out this 
to him. 